\title{
Numerical Studies of Miniband Conduction in Quasi-One-Dimensional Superlattices
}

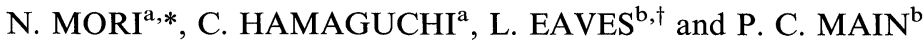 \\ ${ }^{a}$ Department of Electronic Engineering, Osaka University, Suita City, Osaka 565-0871, Japan; \\ ${ }^{\mathrm{b}}$ School of Physics and Astronomy, University of Nottingham, Nottingham NG7 2RD, United Kingdom
}

Monte Carlo simulations of electron motion in GaAs/AlAs superlattices with narrow mini-band width are performed to investigate the effect of a strong magnetic field on miniband conduction. In the quantum limit at low temperatures when the cyclotron energy exceeds the miniband width, the miniband conduction is found to exhibit a strong suppression. This results from the quasi-one-dimensional states formed in the quantum limit and the restricted range of the scattering processes available to the conduction electrons. A small shoulder on the lower electric field side of the main peak is also found in marked contrast with Esaki-Tsu and Ignatov models.

Keywords: Superlattice; GaAs/AlAs; Transport; Monte Carlo simulation; Galvanomagnetic effect; Electron-phonon interaction

\section{INTRODUCTION}

Since the pioneering work of Esaki and Tsu [1], miniband conduction in semiconductor superlattices has been extensively investigated in electrical transport experiments [2]. Miniband conduction in a superlattice is characterized by the presence of a peak and associated negative differential conductance in the current-voltage characteristics. The form and voltage position of the peak are described by Esaki-Tsu [1] and Ignatov [3] models. The peak occurs when $\omega_{\mathbf{B}} \tau=1$ with $\omega_{\mathbf{B}}=e F d / \hbar$, where $\tau$ is some average scattering time, $F$ the electric field applied along the growth direction and $d$ the superlattice period. The Esaki-Tsu and Ignatov models, however, assume simplified scattering processes whose scattering rates are independent of the electron energy. Artaki and Hess [4] and Sibille et al. [5] studied electron transport in GaAs/AlGaAs superlattices by means of Monte Carlo simulation including realistic scattering mechanisms.

In the present work we performed Monte Carlo simulations of electron motion in GaAs/AlAs superlattices with narrow miniband width $E_{\mathrm{B}}<\hbar \omega_{\mathrm{LO}}$ to investigate the effect of a strong magnetic field $B$ on the miniband conduction. The magnetic field is applied perpendicular to the

\footnotetext{
* Corresponding author. Tel.: +8166879 7766, Fax: +8166879 7753, e-mail: mori@ele.eng.osaka-u.ac.jp

${ }^{\dagger}$ Tel.: +44115951 5162, Fax: +441159515180.
} 
planes of the superlattice quantum wells and acts to quantize the in-plane motion into Landau levels. In the quantum limit at low temperatures when the cyclotron energy $\hbar \omega_{c}$ exceeds the miniband width $E_{\mathrm{B}}$, we find a strong suppression of miniband conduction. We show this behavior results from the quasi-one-dimensional states formed in the quantum limit and the restricted range of the scattering processes available to the conduction electrons under these conditions. We also find that the current-voltage characteristics have a small shoulder on the lower electric field side of the main peak in marked contrast with Esaki-Tsu and Ignatov models. We show the shoulder originates from the presence of a threshold in the optic-phonon emission at the opticphonon energy, $\hbar \omega_{\text {LO }}$.

\section{FORMALISM}

\subsection{Electronic States}

We consider a GaAs/AlAs superlattice whose geometry is given in Figure 1. In the superlattice, there are heterointerfaces normal to the $z$ axis, and electric and magnetic fields are applied parallel to the $z$ axis. The natural basis states for electrons at zero electric field are the Landau level miniband (LLMB) states $|\lambda\rangle$ where $\lambda=\{N, X, k\}$ : the set of quantum numbers for the Landau level, the guiding center coordinate and the wavevector along the $z$ direction. The functions

$$
\Psi_{N X k}(\mathbf{r})=\phi_{N}(x-X) \exp \left(-i \frac{X}{\ell^{2}} y\right) u_{k}(z)
$$

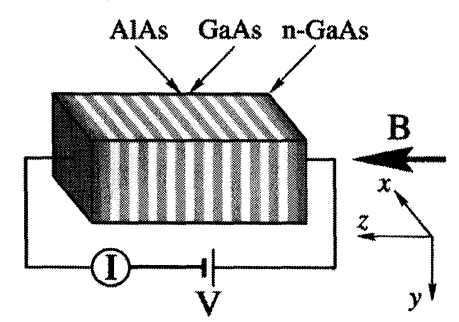

FIGURE 1 Schematic diagram of an undoped GaAs/AlAs superlattice together with the coordinate system. are single-electron eigenstates in the Landau gauge where $\phi_{N}(x-X)$ are the simple harmonicoscillator-like solutions and $\ell=(\hbar / e B)^{1 / 2}$. Within a tight-binding approximation, the superlattice wave-functions $u_{k}(z)$ are given by

$$
u_{k}(z)=\frac{1}{\sqrt{M}} \sum_{\nu} u_{o}(z-\nu d) \mathrm{e}^{i \nu k d}
$$

where $u_{0}(z-\nu d)$ is the wave-function of an eigenstate $E_{0}$ in a given quantum well $\nu$ and $M$ the total number of quantum wells. The energy spectrum of the LLMB states $|\lambda\rangle$ is then written as

$$
E_{N k}=\left(N+\frac{1}{2}\right) \hbar \omega_{\mathrm{c}}+\frac{1}{2} E_{\mathrm{B}}(1-\cos k d)+E_{0}
$$

where $\omega_{\mathrm{c}}=e B / m$ and $E_{\mathrm{B}}$ is the miniband width. We take the electric field into account using the acceleration theorem. Wannier-Stark quantization $[6,7]$ is not included in the following analysis.

\subsection{Scattering Processes}

\subsubsection{Interface-roughness Scattering}

Interface-roughness is caused by change in the quantum well width $w$ and in the superlattice period $d$. The change in $w$ affects electronic states through $E_{0}$, and the change in $d$ affects electronic states through the hopping matrix element $t\left(=E_{\mathrm{B}} / 4\right)$. We take into account only the effect of the change in $w$ because of the thin quantum well width. The scattering potential is then given by

$$
V(x, y, z)=\sum_{\nu} \frac{\partial E_{0}}{\partial w} \Delta_{\nu}(x, y) \Pi_{\nu}(z)
$$

where $\Delta_{\nu}(x, y)$ is the change in the $\nu$-th quantum well width at $(x, y)$ and

$$
\Pi_{\nu}(z)=\left\{\begin{array}{ll}
1 & (z \text { in the } \nu \text {-th quantum well }) \\
0 & \text { (otherwise })
\end{array} .\right.
$$

The roughness $\Delta_{\nu}(\mathbf{R})(\mathbf{R}=(x, y))$ has been assumed to be characterized by the correlation 
function

$$
\left\langle\Delta_{\nu^{\prime}}\left(\mathbf{R}^{\prime}\right) \Delta_{\nu}(\mathbf{R})\right\rangle=\delta_{\nu^{\prime} \nu} \Delta^{2} \exp \left(-\frac{\left.\left|\mathbf{R}^{\prime}-\mathbf{R}\right|^{2}\right)}{\Lambda^{2}}\right)
$$

where $\langle\cdots\rangle$ means an average, $\Delta$ is the meansquare deviation of the change in the well width, and $\Lambda$ the lateral spatial decay rate of the roughness.

The interface-roughness scattering rate can be calculated using Eqs. (5) and (6), and we have

$$
\begin{aligned}
W_{N X k}= & \frac{2 \pi}{\hbar} \sum_{N^{\prime} X^{\prime} k^{\prime}}\left\langle\left|\left(N^{\prime} X^{\prime} k^{\prime}|V(x, y, z)| N X k\right)\right|^{2}\right\rangle \\
& \times \delta\left(E_{N^{\prime} k^{\prime}}-E_{N k}\right) \\
= & \frac{1}{\hbar}\left(\frac{\partial E_{0}}{\partial w} \Delta\right)^{2}\left(\frac{\Lambda}{\ell}\right)^{2} \frac{2}{E_{\mathrm{B}}} \sum_{N^{\prime}} \frac{L_{N N^{\prime}}}{\sqrt{1-p_{\mathrm{IR}}^{2}}},
\end{aligned}
$$

where

$$
\begin{gathered}
L_{N N^{\prime}}=\int_{0}^{\infty} J_{N N^{\prime}}^{2}(\zeta) \exp \left(-\frac{\Lambda^{2}}{2 \ell^{2}} \zeta\right) d \zeta \\
p_{\mathrm{IR}}=\left(N^{\prime}-N\right) \frac{2 \hbar \omega_{\mathrm{c}}}{E_{\mathrm{B}}}+\cos k d
\end{gathered}
$$

and $J_{N N^{\prime}}(\zeta)$ are the matrix elements between the simple harmonic-oscillator-like solutions [8].

\subsubsection{Polar-optic-phonon Scattering}

For bulk longitudinal optic-phonons with Fröhlich polar coupling potential $C_{\text {pop }}(q)$, the polar-optic-phonon scattering rate is given by (the upper/lower sign is for emission/absorption of a phonon)

$$
\begin{aligned}
W_{N X k}^{ \pm}= & \frac{2 \pi}{\hbar} \sum_{N^{\prime} X^{\prime} k^{\prime}} \sum_{\mathbf{q}}\left|C_{\mathrm{POP}}(q)\right|^{2}\left|\left(N^{\prime} X^{\prime} k^{\prime}\left|\mathrm{e}^{i \mathbf{q} \cdot \mathbf{r}}\right| N X k\right)\right|^{2} \\
& \times\left(N_{0}+\frac{1}{2} \pm \frac{1}{2}\right) \delta\left(E_{N^{\prime} k^{\prime}}-E_{N k} \pm \hbar \omega_{L O}\right) \\
= & 4 \alpha \omega_{\mathrm{LO}}\left(N_{0}+\frac{1}{2} \pm \frac{1}{2}\right) \frac{1}{k_{0} w} \frac{\hbar \omega_{\mathrm{LO}}}{E_{\mathrm{B}}} \sum_{N^{\prime}} \frac{M_{N N^{\prime}}}{\sqrt{1-p_{\mathrm{POP}}^{2}}}
\end{aligned}
$$

where

$$
\begin{gathered}
M_{N N^{\prime}}=\int_{0}^{\infty} F(Q) J_{N N^{\prime}}^{2}\left(\frac{1}{2} l^{2} Q^{2}\right) w d Q, \\
F(Q)=\iint u_{0}^{2}(z) e^{-Q\left|z-z^{\prime}\right|} u_{0}^{2}\left(z^{\prime}\right) d z d z^{\prime}, \\
p_{\text {POP }}=\frac{2}{E_{B}}\left\{\left(N^{\prime}-N\right) \hbar \omega_{\mathrm{c}} \pm \hbar \omega_{\mathrm{LO}}\right\}+\cos k d,
\end{gathered}
$$

$\alpha$ is Fröhlich coupling constant, $\hbar w_{\text {LO }}$ the opticphonon energy, $\mathrm{N}_{0}$ the optic-phonon occupation number, and $k_{0}=\left(2 m \omega_{\mathrm{LO}} / \hbar\right)^{1 / 2}$.

\subsubsection{Acoustic-deformation Scattering}

We take into account of deformation potential coupling to bulk acoustic-phonons with the interaction potential

$$
\left|C_{\mathrm{ADP}}(q)\right|^{2}=\frac{D^{2}}{2 \rho s_{l}^{2}} \hbar \omega_{q}
$$

with $D$ being the deformation potential, $\rho$ the density of GaAs, $\mathrm{s}_{l}$ the longitudinal sound velocity, and $\hbar \omega_{q}$ the acoustic-phonon energy. The acousticdeformation scattering rate is calculated in a similar way to the optic-phonon scattering rate.

\subsubsection{Piezoelectric Scattering}

Piezoelectric coupling to acoustic-phonons is taken into account in the same manner in Ref. [9]. The interaction potential is given by

$$
\left|C_{\mathrm{PEL}}(q)\right|^{2}=\frac{\left(e h_{14}\right)^{2}}{2 \rho s_{l}^{2}} \frac{A_{l}}{q^{2}} \hbar \omega_{q}+\frac{\left(e h_{14}\right)^{2}}{2 \rho s_{t}^{2}} \frac{2 A_{t}}{q^{2}} \hbar \omega_{q}
$$

where $h_{14}$ is the basic piezoelectric tensor component and $s_{t}$ the transverse sound velocity. The coefficients $A_{1}$ and $A_{t}$ depend strongly on the direction of phonon wavevector [9]. The piezoelectric scattering rate is calculated in a similar way to the optic-phonon scattering rate. 


\subsection{Monte Carlo Simulation}

We consider only the low electron density case and the drift-velocity $v_{\text {drift }}$ is calculated as a function of applied electric and magnetic fields by means of single-electron Monte Carlo simulations of the electron motion in LLMBs. In the case of high electron density, the frequency of mutual collisions of electrons is larger than the frequency of electron-lattice collisions and it may be adequate to adopt other methods $[10,11]$ that express the distribution function in terms of macroscopic variables such as effective electron temperature, drift-velocity, and chemical potential.

\section{RESULTS AND DISCUSSION}

Figure 2 shows calculated $v_{\text {drift }}$-eFd characteristics of a GaAs/AlAs superlattice with $d=10.6 \mathrm{~nm}$ and $w=9.3 \mathrm{~nm}$ at $T=4.2 \mathrm{~K}$ and $B=5 \mathrm{~T}$. The miniband width $E_{\mathrm{B}}=10 \mathrm{meV}$ is estimated by modeling the superlattice with the effective mass approximation. We assume an infinite square quantum well to calculate the electronic state, $u_{0}(\mathrm{z})$ and $E_{0}$, for each quantum well. Scattering rates are evaluated using the material parameters of bulk GaAs. For the interface roughness scattering, we set $\Delta=0.28 \mathrm{~nm}$ and $\Lambda=5 \mathrm{~nm}$.

The simulations yield a $v_{\text {drift }}(F)$ curve similar to the Esaki-Tsu form except for a small shoulder at

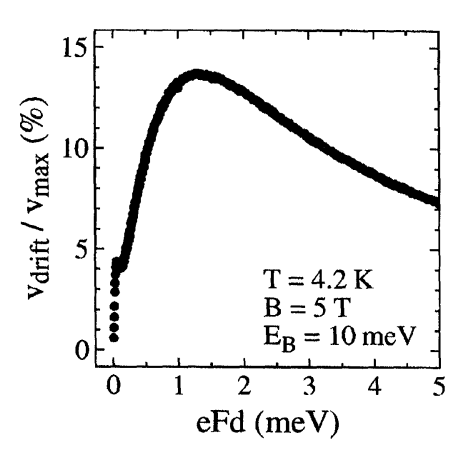

FIGURE 2 Drift-velocity $v_{\text {drift }}$ as a function of Bloch energy $e F d$ for a GaAs/AlAs superlattice with $d=10.6 \mathrm{~nm}$ and $w=$ $9.3 \mathrm{~nm} . v_{\text {drift }}$ is normalized by the maximum velocity $v_{\max }=$ $E_{\mathrm{B}} d / 4 \hbar$ of the miniband. $\mathrm{e} F d \sim 0.05 \mathrm{meV}$ (whose origin is discussed later). However, the peak value of $v_{\text {drift }}$ is much smaller than the maximum velocity $v_{\max }=E_{\mathrm{B}} d / 4 \hbar$ of the miniband, as expected for the general case where both elastic and inelastic scattering processes are present [3]. As shown in Figure 3 where we plot the magnetic field dependence of $v_{\text {drift }}$ at $e F d=$ $1.5 \mathrm{meV}$ and $T=4.2 \mathrm{~K}$, the drift-velocity has a weak oscillatory variation with $B$ below $6 \mathrm{~T}$. The minima are associated with the conditions (i) $n \hbar \omega_{c}=E_{B}$ or (ii) $n \hbar \omega_{\mathrm{c}}+E_{\mathrm{B}}=\hbar \omega_{\mathrm{LO}}(n=$ $1,2,3, \ldots)$. When condition (i) is satisfied, the interface-roughness scattering is enhanced due to alignment of the maximum of the $(n+r)^{\text {th }}$ LLMB with the maximum of the $r^{\text {th }}$ LLMB. On the other hand, the optic-phonon scattering is enhanced for magnetic fields higher than the condition (ii) because electrons in the $(n+r)^{\text {th }}$ LLMB can relax into the $r^{\text {th }}$ LLMB via optic-phonon emission. The most striking feature of the simulation is the strong quenching of the drift-velocity at about $6 \mathrm{~T}$.

In order to explain the quenching of the driftvelocity, the electron motion in LLMBs is shown schematically in Figure 4 for two distinct regimes of magnetic fields $\hbar \omega_{\mathrm{c}}<E_{\mathrm{B}}$ and $\hbar \omega_{\mathrm{c}}>E_{\mathrm{B}}$. At low $T$ and low $B$, when $\hbar \omega_{\mathrm{c}}<E_{\mathrm{B}}$ (Fig. 4a), electrons can emit optic-phonons because an electron accelerated by applied electric field is elastically scattered into higher LLMBs, for example as shown by the line from $\alpha$ to $\beta$ in Figure 4(a),

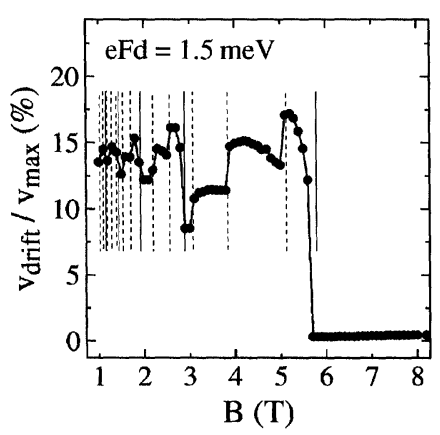

FIGURE 3 Magnetic field dependence of the drift-velocity $v_{\text {drift }}$ at $e F d=1.5 \mathrm{meV}$. Vertical solid lines show the condition of $n \hbar \omega_{\mathrm{c}}=E_{\mathrm{B}}$ and vertical dashed lines the condition of $n \hbar \omega_{\mathrm{c}}+E_{B}=\hbar \omega_{\mathrm{LO}}(n=1,2,3, \ldots)$. The lines connecting solid circles are guides to the eye only. 

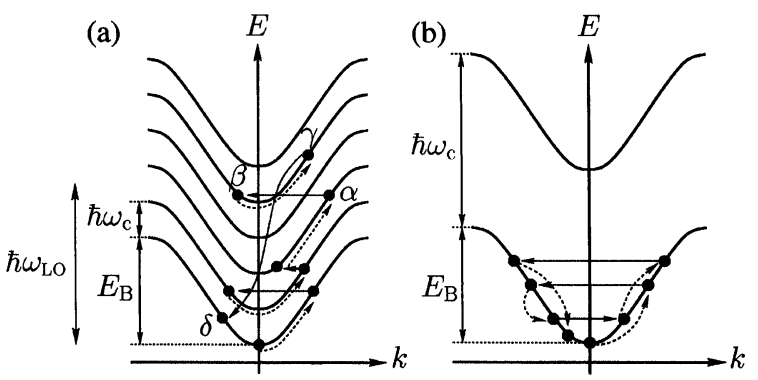

FIGURE 4 Schematic energy dispersion relation showing possible electron motion at low temperatures. (a) for $\hbar \omega_{\mathrm{c}}<E_{\mathrm{B}}$ : arrows from $\alpha$ to $\beta, \beta$ to $\gamma$, and $\gamma$ to $\delta$ represent interface-roughness scattering, free flight of the electron, and optic-phonon emission, respectively. (b) for $\hbar \omega_{\mathrm{c}}>E_{\mathrm{B}}$ where the electron is confined to the lowest Landau level miniband.

and subsequently gains the kinetic energy higher than $\hbar \omega_{\text {LO }}$. The energy relaxation via optic-phonon emission is so efficient that the $v(F)$ curves exhibit Ohmic behavior and the drift-velocity increases with $F$ for $e F d \lesssim 1 \mathrm{meV}$ before the system enters the negative differential conductance (NDC) regime. At high $B$ when $\hbar \omega_{\mathrm{c}}>E_{\mathrm{B}}$ (Fig. 4b), on the other hand, the LLMBs are effectively decoupled and the electron undergoes interfaceroughness and acoustic-phonon scatterings within the $N=0$ LLMB because $E_{\mathrm{B}} \ll \hbar \omega_{\mathrm{LO}}$. The electron-acoustic-phonon interaction is weak and the system enters the NDC regime at $e F d \sim$ $0.05 \mathrm{meV}$. Above this field, the electron is constrained to move and to scatter mainly by the interface-roughness scattering between states of equal and opposite $k$ within the $N=0$ LLMB. The center of the distribution function therefore remains at $\mathrm{k} \sim 0$, leading to the strong suppression of miniband conduction under these conditions.

Next we discuss the origin of the small shoulder at $e F d \sim 0.05 \mathrm{meV}$ in the $v(F)$ curve. Electrons relax their energy via acoustic-phonon emission for $e F d \lesssim 0.05 \mathrm{meV}$. The electron can dissipate any small amount of energy to acoustic-phonons because of the linear dispersion relation of the acoustic-phonon. The system therefore shows normal Ohmic behavior

$$
v_{\mathrm{drift}}=\frac{e \tau_{\mathrm{m}}}{m_{\mathrm{SL}}} F
$$

with the miniband-edge effective mass $m_{\mathrm{SL}}=$ $\left[\left.\left(1 / \hbar^{2}\right)\left(\partial^{2} E_{k} / \partial k^{2}\right)\right|_{k=0}\right]^{-1}$ and the momentum relaxation time $\tau_{\mathrm{m}}$ due to the interface-roughness scattering. This Ohmic behavior for $e F d \lesssim$ $0.05 \mathrm{meV}$ does not exhibit a sudden change at $\hbar \omega_{\mathrm{c}}=E_{\mathrm{B}}$ (see Fig. 5) because the optic-phonon emission is irrelevant to the energy relaxation in this linear response regime. At high $F$, when $e F d \gtrsim 0.05 \mathrm{meV}$, electrons cannot dissipate all the energy to acoustic-phonons and electron heating occurs resulting in two distinct behavior of $v(F)$ curves depending on $B$. At high $B$, when $\hbar \omega_{\mathrm{c}}>E_{\mathrm{B}}$, a strong suppression of miniband conduction occurs as explained in the previous paragraph. At low $B$, when $\hbar \omega_{\mathrm{c}}<E_{\mathrm{B}}$, on the other hand, an electron becomes possible to relax its energy via optic-phonon emission once the kinetic energy exceeds $\hbar \omega_{\text {Lo }}$ through the inter LLMBs scatterings. The system then shows the second Ohmic behavior $v_{\text {drift }}=\left(e \tau_{\mathrm{m}} / M_{\mathrm{SL}}\right) F$ with a superlattice effective mass $M_{\mathrm{SL}}$ which includes effects of the electron heating and is heavier than the miniband-edge effective mass $m_{\mathrm{SL}}$. These features can be readily seen in Figure 5 where we plot the $v(F)$ curves calculated with and without acousticphonon scatterings at $B=5 \mathrm{~T}$. The small shoulder in the $v(F)$ curves is therefore attributed to the difference between $m_{\mathrm{SL}}$ and $M_{\mathrm{SL}}$ and to the fact that the optic-phonon emission has a threshold at the optic-phonon energy $\hbar \omega_{\text {LO }}$.

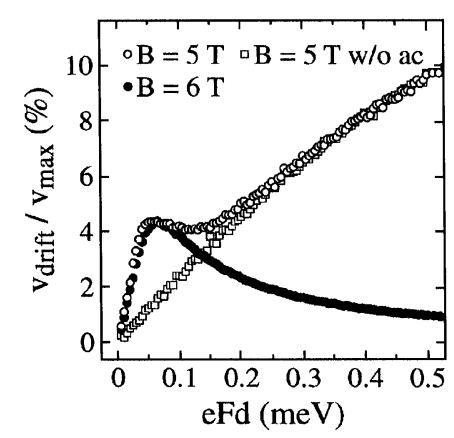

FIGURE 5 Drift-velocity $v_{\text {drift }}$ as a function of $e F d$ for low electric field region at $B=5 \mathrm{~T}$ (open circles) and $B=6 \mathrm{~T}$ (closed circles). Open squares show $v_{\text {drift }}$ calculated without acousticphonon scatterings at $B=5 \mathrm{~T}$. 


\section{References}

[1] Esaki, L. and Tsu, R. (1970). "Superlattice and Negative Differential Conductivity in Semiconductors", IBM Journal of Research and Development, 14, 61-65.

[2] Semiconductor Superlattices (1995). edited by Grahn, H. T., Singapore: World Scientific.

[3] Ignatov, A. A., Dodin, E. P. and Shashkin, V. I. (1991). "Transport Response Theory of Semiconductor Superlattices: Connection with Bloch Oscillations", Modern Physics Letters, 5, 1087-1094.

[4] Artaki, M. and Hess, K. (1985). "Monte Carlo Calculations of Electron Transport in GaAs/AlGaAs Superlattices", Semiconductor and Mictrostructures, 1, $489-493$.

[5] Sibille, A., Palmier, J. F., Hadjazi, M., Wang, H., Etemadi, G., Dutisseuil, E. and Mollot, F. (1993). "Limits of Semiclassical Transport in Narrow Miniband GaAs/
AlAs Superlattices", Semiconductor and Mictrostructures, 13, 247-253.

[6] Tsu, R. and Döhlier, G. (1975). "Hopping Conduction in a 'Superlattices"', Physical Review B, 12, 680-686.

[7] Wacker, A. and Jauho, A.-P. (1999). "Inelastic Quantum Transport in Superlattices: Success and Failure of the Boltzmann Equation", Physical Review Letters, 83, 836839.

[8] Kubo, R., Miyake, S. J. and Hashitume, N. (1964). Solid State Physics, Academic Press: New York, 17, 269-364.

[9] Price, P. J. (1981). "Two-dimensional Electron Transport in Semiconductor Layers. I. Phonon Scattering", Annals of Physics, 133, 217-239.

[10] Zubarev, D. N., Nonequilibrium Statistical Thermodynamics, Prenum: New York, 1974

[11] Shu, W. M. and Lei, X. L. (1994). "Miniband Transport in Semiconductor Superlattices in a Quantized Magnetic Field", Physical Review B, 50, 17378-17382. 

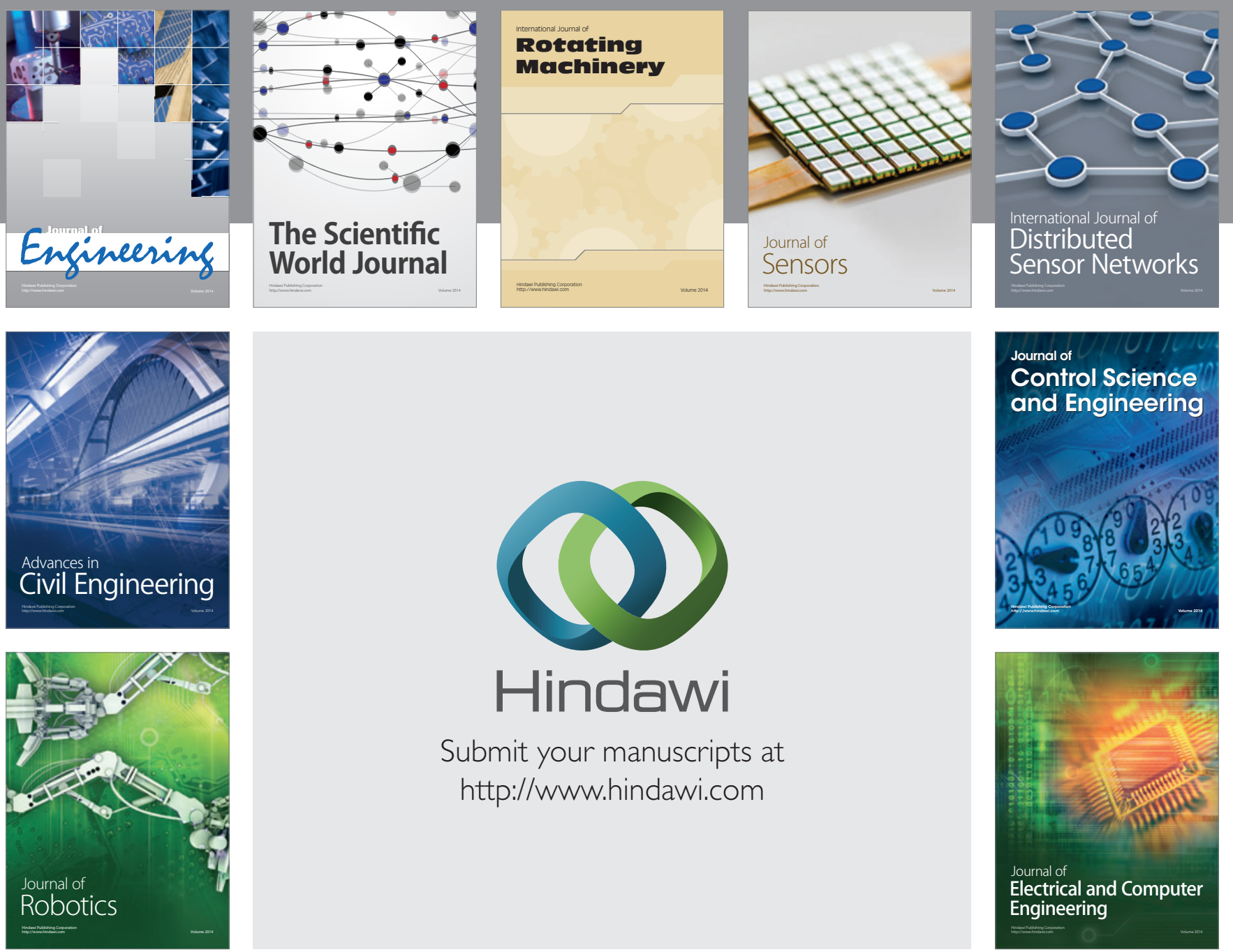

Submit your manuscripts at

http://www.hindawi.com
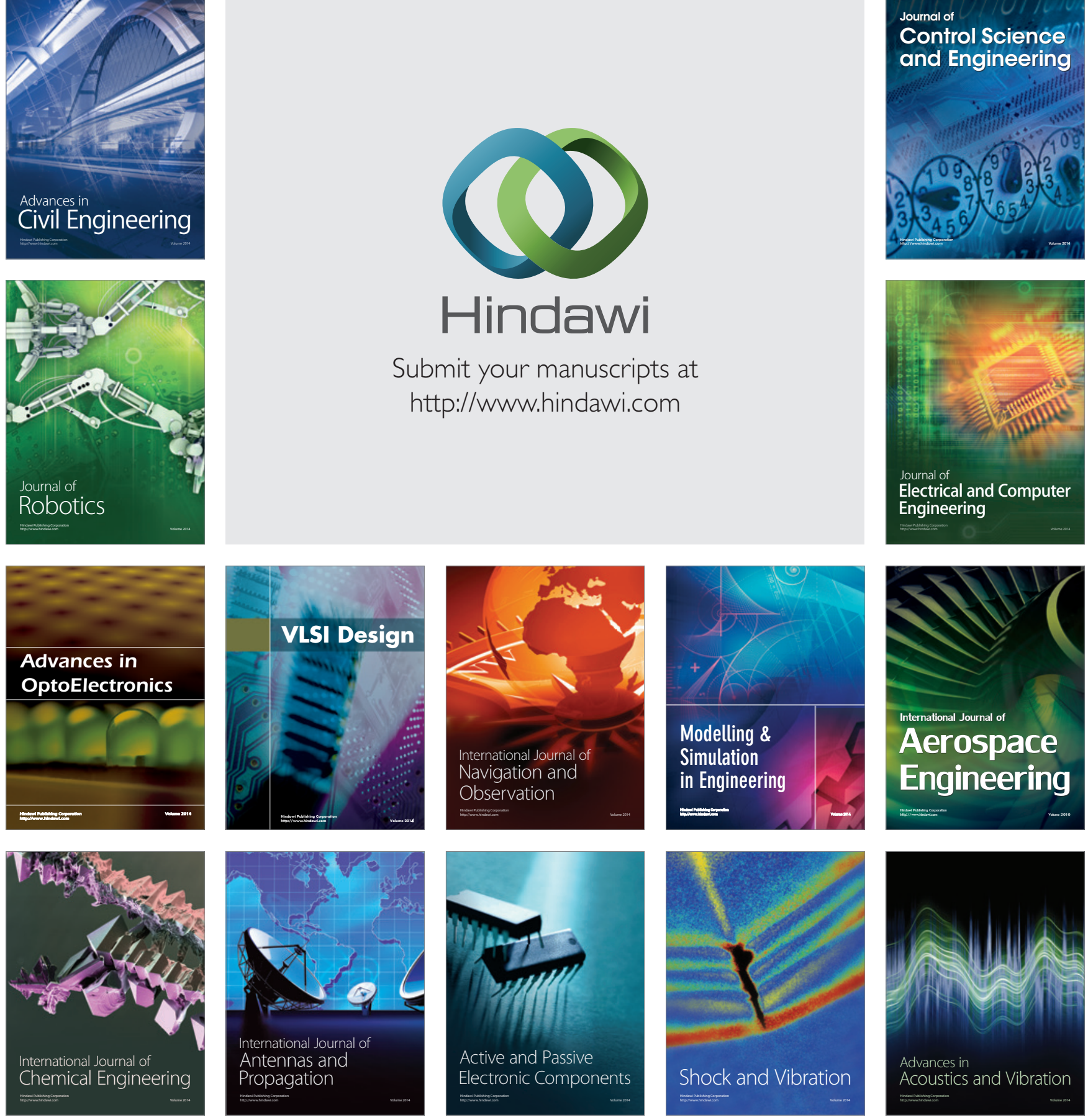